\title{
A Jost-Schroer Theorem for String Fields
}

\author{
O. Steinmann
}

Fakultät für Physik, Universität Bielefeld, D-4800 Bielefeld 1, Federal Republic of Germany

\begin{abstract}
It is shown that the truncated Wightman functions of three or more string-localized fields vanish if they are solutions of a Klein-Gordon equation in each variable. As an application it is shown that a string field is a free field if its two-point functions are those of a free field. Another application to perturbation theory is pointed out.
\end{abstract}

\section{Introduction, Results}

Gauge theories confront us with, among others, the following two problems.

i) There exist very different looking but physically equivalent formulations, called "gauges," of the theory. This makes the interpretation of the formalism, in particular the search for the physical significance of the basic fields, difficult.

ii) In the physical gauges, i.e. formulations which work in a physical state space with positive metric, the basic fields are neither covariant nor local. As a consequence, many of the well-known methods and results of local field theory are not immediately applicable. This holds especially for most of the rigorous results of axiomatic field theory.

Mandelstam [1] proposed to solve the first of these problems, e.g. in QED, by working only with the gauge independent fields $F_{\mu \nu}(x)$ and $\psi(x) \exp \left\{-i e \int_{-\infty}^{x} d \xi^{\mu} A_{\mu}(\xi)\right\}$. Since this device merely replaces gauge dependence by path dependence the gain might look doubtful at first. However, recently Buchholz and Fredenhagen [2] have shown that this strategy also goes a long way towards solving problem ii): important axiomatic results can be derived for fields which are localized on space-like strings or more generally in space-like cones. In particular this is true for the construction of asymptotic scattering states, i.e. for the very nontrivial problem of identifying states of the formalism with the states found in nature. Further investigation of charged string-localized fields is thus indicated, continuing both the rigorous approach of Buchholz and Fredenhagen as well as the more down-to-earth dynamical studies of specific models as carried out by Mandelstam and others $[1,3]$. 
In the present work we prove a theorem which is useful in both respects, leading on the one hand to a generalization of the Jost-Schroer theorem of local field theory, having on the other hand an important application in perturbation theory.

Let $\psi_{i}(x), i=1, \ldots, N$, be fields satisfying the Wightman axioms (see e.g. [4]) with the following modifications.

1) Invariance under the homogeneous Lorentz group is not assumed.

2) Locality holds only in a weakened form ("string locality"): let $e$ be a spacelike unit vector, which we choose (without restriction of generality) as $e=(0,0,0,1)$. Let $M$ be the four-dimensional Minkowski space and define, for $x \in M$, the string

We assume

$$
S_{x}=\{z \in M: z=x+\varrho e, \varrho \geqq 0\} .
$$

$$
\left[\psi_{i}(x), \psi_{j}(y)\right]_{ \pm}=0
$$

if $S_{x}$ and $S_{y}$ are totally space-like relative to each other. The commutator or anticommutator occurs as usual according to the statistics of the fields involved. Condition (1.2) will be called string-locality. Note that the string direction $e$ is assumed to be the same for all fields.

The Wightman functions $W\left(x_{1}, \ldots, x_{n}\right)=\left(\Omega, \psi_{i_{1}}\left(x_{1}\right) \ldots \psi_{i_{n}}\left(x_{n}\right) \Omega\right)$ have then the well-known properties: temperedness, translational invariance, $p$-space support, string locality, and the cluster property which we use in the following form. For $W$ we can write down the familiar cluster expansion

$$
W\left(x_{1}, \ldots, x_{n}\right)=\sum \pm W^{T}\left(x_{1}, \ldots\right) \ldots W^{T}\left(\ldots, x_{n}\right) .
$$

The Fourier transform $\tilde{W}^{T}\left(p_{1}, \ldots, p_{n}\right)$ of the truncated Wightman function $W^{T}\left(x_{1}, \ldots, x_{n}\right)$ is, after integration over test functions in $p_{1}, \ldots, p_{\alpha-1}, p_{\alpha+1}, \ldots, p_{n}$, $\alpha<n$, a measure in the variable $P_{\alpha}=p_{1}+\ldots+p_{\alpha}$ with no point contribution at $P_{\alpha}=0$, so that $\tilde{W}^{T}$ is uniquely determined by its values on test functions which vanish at $P_{\alpha}=0$ with all their derivatives.

The positivity property of the $W$ will be used for the proof of the Jost-Schroer theorem (Theorem 2) but not for the proof of Theorem 1, the main result of this paper.

We now state this result:

Theorem 1. Let $W\left(x_{1}, \ldots, x_{n}\right)$ be Wightman functions with the modified properties as stated above, positivity not being assumed. Assume

for all $i$, with $m_{i} \geqq 0$. Then

$$
\left(\square_{i}+m_{i}^{2}\right) W^{T}\left(x_{1}, \ldots, x_{n}\right)=0
$$

for $n \geqq 3$.

$$
W^{T}\left(x_{1}, \ldots, x_{n}\right)=0
$$

The proof of this theorem will be given in Sect. 2 .

Remarks. 1) In QED the $\psi_{i}$ are the components of $F_{\mu \nu}$ and of the Mandelstam string fields $\psi, \bar{\psi}$. The $F_{\mu \nu}$ are local in the strict point-wise sense, and using this the proof of Theorem 1 can be somewhat simplified. However, strict locality implies string locality. and our proof is preferable because it applies also to non-abelian gauge theories containing massless string fields. 
2) If string localization is replaced by the cone localization of Buchholz and Fredenhagen, then Theorem 1 cannot be proved if the cones are the same for all variables. It can be proved, however, if the cones are allowed to depend on $i$, and are chosen such that the set $C_{i}-C_{k}$ is space-like for all $i \neq k$. Here $C_{i}$ is the cone associated with $x_{i}$, with its apex lying at the origin.

We conclude this section with two applications of Theorem 1 . The first one is a generalization of the Jost-Schroer theorem [5] to string-localized fields.

Theorem 2. Let $\psi(x)$ be a field satisfying the modified Wightman axioms including positivity. Assume its two-point functions to be the two-point functions of a local free field of mass $m \geqq 0$, so that

$$
\left(\square_{y}+m^{2}\right)\left(\Omega, \psi(x) \psi^{*}(y) \Omega\right)=\left(\square_{y}+m^{2}\right)\left(\Omega, \psi^{*}(x) \psi(y) \Omega\right)=0 .
$$

Then $\psi$ is a local free field.

The word "local" is here used in the strict sense of point locality.

Proof of Theorem 2. Using positivity we obtain from (1.5):

$$
\left(\square+m^{2}\right) \psi(x) \Omega=\left(\square+m^{2}\right) \psi^{*}(x) \Omega=0 .
$$

Since the usual spectral properties are assumed, $W^{T}\left(x_{1}, \ldots, x_{n}\right)$ is boundary value of an analytic function in the forward tube $\left\{\operatorname{Im}\left(x_{i+1}-x_{i}\right) \in V_{+}\right.$for $\left.i=1, \ldots, n-1\right\}$. (Here $x_{i}$ is a $\psi$ - or a $\psi^{*}$-variable, as the case may be.) The same analyticity property holds then for

$$
F_{i}\left(x_{1}, \ldots, x_{n}\right)=\left(\square_{i}+m^{2}\right) W^{T}\left(x_{1}, \ldots, x_{n}\right) .
$$

Let $B$ be the unit ball in $M$ with centre at the origin, $B_{a}$ the unit ball with centre at $(0,0, a, 0), a$ sufficiently large. If $x_{i} \in B_{a}, x_{i+1}, \ldots, x_{n} \in B$, then $S_{x_{i}}$ is totally space-like relative to all $S_{x_{j}}$ with $j>i$, hence by string locality:

$$
F_{i}\left(x_{1}, \ldots, x_{n}\right)=\left(\square_{i}+m^{2}\right) W^{T}\left(\ldots, x_{i-1}, x_{i+1}, \ldots, x_{n}, x_{i}\right)=0
$$

by (1.6). This is true in an open set in $M^{n}$, and thus $F_{i} \equiv 0$ by analytic continuation. This means that the assumptions of Theorem 1 are satisfied, hence $W^{T} \equiv 0$ for $n \geqq 3$. This cluster expansion of $W\left(x_{1}, \ldots, x_{n}\right)$ contains then only two-point functions, and these are free by assumption: the $W$ are the Wightman functions of a free field, hence $\psi$ is free.

The second application of Theorem 1 is to perturbation theory formulated in terms of Wightman functions. Such a formulation has certain advantages over the more familiar one using Green's functions : firstly it avoids the problems connected with the ambiguity of the T-product at coinciding times and, secondly, no equaltime commutation relations are needed. The formalism will be described elsewhere for the case of QED. Here we only mention the point at which Theorem 1 comes in.

In a field theory amenable to perturbation methods the $\sigma^{\text {th }}$ order term $W_{\sigma}$ in the perturbative expansion of $W$ is determined recursively as solution of a system of differential equations

$$
D_{i} W_{\sigma}\left(\ldots, x_{i}, \ldots\right)=I_{\sigma i}\left(\ldots, x_{i}, \ldots\right)
$$

where $D_{i}$ is a differential operator, e.g. $\left(\square+m^{2}\right),(\not \partial+m)$, or simply $\partial_{v}$, and where the right-hand side $I_{\sigma i}$ is known if the $W_{\tau}$ with $\tau<\sigma$ are known. In all the familiar 
models Eq. (1.8) imply equations of the form

$$
\left(\square_{i}+m_{i}^{2}\right) W_{\sigma}^{T}\left(\ldots, x_{i}, \ldots\right)=I_{\sigma i}^{\prime}\left(\ldots, x_{i}, \ldots\right),
$$

where again $I_{\sigma i}^{\prime}$ is completely determined by the lower orders of perturbation theory. Hence $W_{\sigma}^{T}$ is determined uniquely up to a solution of the homogeneous equations $\left(\square_{i}+m_{i}^{2}\right) \Delta_{\sigma}^{T}\left(x_{1}, \ldots, x_{n}\right)=0$. But according to Theorem 1 these equations have for $n \geqq 3$ no non-trivial solutions satisfying the modified Wightman conditions. Hence $W^{T}\left(x_{1}, \ldots, x_{n}\right), n>2$, is determined uniquely if we demand that it satisfy these conditions, with the exception of positivity which is beyond the powers of perturbation theory. The ambiguities of the two-point functions are removed by imposing suitable renormalization conditions.

\section{Proof of Theorem 1}

The Fourier transform of any function or distribution $F$ will be denoted by $\tilde{F}$. Equation (1.3) implies

hence

$$
\left(p_{i}^{2}-m_{i}^{2}\right) \tilde{W}^{T}\left(p_{1}, \ldots, p_{n}\right)=0
$$

$$
\operatorname{supp} \tilde{W}^{T}\left(p_{1}, \ldots, p_{n}\right) \subset\left\{p_{i}^{2}=m_{i}^{2}, \quad \forall i\right\} .
$$

Due to the spectral condition the $p_{1}$-support lies on the positive mass shell $p_{1}^{2}$ $=m_{1}^{2}, p_{10} \geqq 0$.

Define

$$
\begin{gathered}
X=x_{1}+x_{2}, \quad \xi=x_{1}-x_{2}, \quad P=\frac{1}{2}\left(p_{1}+p_{2}\right), \quad Q=\frac{1}{2}\left(p_{1}-p_{2}\right), \\
V_{12}\left(X, \xi, x_{3}, \ldots, x_{n}\right)=W^{T}\left(x_{1}, \ldots, x_{n}\right), \\
V_{21}\left(X, \xi, x_{3}, \ldots, x_{n}\right)= \pm W^{T}\left(x_{2}, x_{1}, x_{3}, \ldots, x_{n}\right) .
\end{gathered}
$$

Here the negative sign applies if both $\psi_{i_{1}}$ and $\psi_{i_{2}}$ are Fermi fields, the positive sign in all other cases. By the spectral condition both $\tilde{V}_{i j}\left(P, Q, p_{3}, \ldots, p_{n}\right)$ have their support in $P \in \bar{V}_{+}$, and by the cluster property it suffices to consider the points with $P \neq 0$.

Consider the $Q$-support of $\tilde{V}_{12}$ for fixed $p_{3}, \ldots, p_{n}$ and fixed $P \in \bar{V}_{+}, P \neq 0$. Its restriction to $p_{20} \geqq 0$ is compact. In $p_{20} \leqq 0$ we have the support conditions $(P+Q)^{2}=m_{1}^{2}, P_{0}+Q_{0} \geqq 0,(P-Q)^{2}=m_{2}^{2}, P_{0}-Q_{0} \leqq 0$. This part of the $Q$-support is also compact if $P^{2}>0$, or if $P^{2}=0$ and at least one of the masses $m_{1}, m_{2}$, is nonzero. If $P^{2}=0$ and $m_{1}=m_{2}=0$, then the support is contained in the half-line $Q=\lambda P, \lambda \geqq 0$. Similar considerations apply to $\tilde{V}_{21}$.

Let $\tilde{\varphi}\left(P, p_{3}, \ldots, p_{n}\right) \in \mathscr{D}$, the space of $C^{\infty}$-functions with compact support. Assume that the $P$-support of $\tilde{\varphi}$ is contained either in the set $\left\{P^{2}>0\right\}$ or in a sufficiently small neighbourhood, not containing the origin, of a light-like point. These $\tilde{\varphi}$ are total in the space of relevant test functions. The support of

$$
\tilde{H}_{+}(Q)=\int d^{4} P \prod_{i=3}^{n} d^{4} p_{i} \tilde{V}_{12}(P, Q, \ldots) \tilde{\varphi}(P, \ldots)
$$

lies in the union of a compact set and a narrow cone $C_{+}$with apex at 0 around a positive light-like direction. In the same way the support of

$$
\tilde{H}_{-}(Q)=\int d^{4} P \prod_{i=3}^{n} d^{4} p_{i} \tilde{V}_{21}(P, Q, \ldots) \tilde{\varphi}(P, \ldots)
$$


is contained in the union of a compact set and the narrow cone $C_{-}=-C_{+}$around a negative light-like direction. The Fourier transforms

$$
H_{ \pm}(\xi)=\int d^{4} X \prod_{3}^{n} d^{4} x_{i} V_{a b}\left(X, \xi, x_{3}, \ldots\right) \varphi\left(X, x_{3}, \ldots\right)
$$

are then boundary values of functions $H_{ \pm}(\zeta)$ analytic in the tubes [6]

$$
\begin{gathered}
\mathscr{T}\left(H_{ \pm}\right)=\left\{\zeta=\xi+i \eta: \eta \in \mathscr{B}_{ \pm}\right\}, \\
\mathscr{B}_{ \pm}=\left\{\eta: \eta Q \lessgtr 0 \text { for all } Q \in\left(C_{+} \backslash 0\right)\right\} .
\end{gathered}
$$

It is important to note that the basis $\mathscr{B}_{+}$contain space-like points. It will be convenient to assume that $C_{+} \backslash 0$ lies fully in one of the open half spaces $Q_{2} \gtrless 0$. For sufficiently narrow $C_{+}$, i.e. for sufficiently small support of $\tilde{\varphi}$, this can always be achieved by a Lorentz transformation in the 0 -2-plane which does not change the direction $e$ of the strings. Then $\mathscr{B}_{+}$contains either the positive or the negative 2-axis.

By string locality $H_{+}$and $H_{-}$coincide in the real domain

$$
\mathscr{R}=\left\{\xi: \xi_{0}^{2}-\xi_{1}^{2}-\xi_{2}^{2}<0\right\} .
$$

The edge-of-the-wedge theorem [7] tells us that $H_{ \pm}(\zeta)$ are analytic continations of each other and that the function $H(\zeta)$ which they define is analytic in

$$
\mathscr{A}=\mathscr{T}\left(H_{+}\right) \cup \mathscr{T}\left(H_{-}\right) \cup \mathscr{N}(\mathscr{R})
$$

where $\mathscr{N}(\mathscr{R})$ is an open complex neighbourhood of $\mathscr{R}$.

Hold $\xi_{0}, \xi_{1}, \xi_{3}$, fixed at some real values, and let $\xi_{2}$ be a complex variable. Then, if $\operatorname{Im} \xi_{2} \neq 0, \xi$ lies either in $\mathscr{T}\left(H_{+}\right)$or in $\mathscr{T}\left(H_{-}\right)$, hence in $\mathscr{A}$. If $\xi_{2}$ is real, then the straight line in the 2-direction through $\left(\xi_{0}, \xi_{1}, 0, \xi_{3}\right)$ meets $C \mathscr{R}$ in a compact set (if $\xi_{0}^{2}-\xi_{1}^{2} \geqq 0$ ) or not at all (if $\xi_{0}^{2}-\xi_{1}^{2}<0$ ). ${ }_{1}^{1}$ In the latter case $\xi$ is in $\mathscr{A}$ for all $\xi_{2}$. If we start from this region and change $\xi_{0}, \xi_{1}$, until our line meets the boundary of $\mathscr{R}$, and thus of $\mathscr{A}$, it will do so in a compact real set in the complex $\xi_{2}$-plane. This is the situation dealt with in the continuity theorem [8], and this theorem tells us that $H(\zeta)$ can be continued analytically into an open neighbourhood of the said compact set. In this way it can be shown that the envelope of holomorphy of $\mathscr{A}$ contains the full real Minkowski space, so that $H_{+}(\xi)=H_{-}(\xi)$ for all $\xi \in M$. But this means

$$
W^{T}\left(x_{1}, x_{2}, \ldots\right)= \pm W^{T}\left(x_{2}, x_{1}, \ldots\right)
$$

Starting from this result we prove by induction that $W^{T}$ is invariant, up to a fermion sign, under all permutations of its arguments. Let $W^{T}$ be invariant under permutations of $x_{1}, \ldots, x_{\alpha}, \alpha \geqq 2$. Define $Y=\frac{1}{2}\left(x_{\alpha}+x_{\alpha+1}\right), y=x_{\alpha+1}-x_{\alpha}$, and

$$
\begin{gathered}
K_{+}\left(x_{1}, \ldots, x_{\alpha-1}, Y, y, x_{\alpha+2}, \ldots\right)=W^{T}\left(x_{1}, \ldots, x_{n}\right), \\
K_{-}\left(x_{1}, \ldots, x_{\alpha-1}, Y, y, x_{\alpha+2}, \ldots\right)= \pm W^{T}\left(x_{1}, \ldots, x_{\alpha+1}, x_{\alpha}, \ldots, x_{n}\right),
\end{gathered}
$$

the sign alternative as in (2.4). After integration over a test function $\varphi(y) \in \mathscr{D}$ both $K_{+}$and $K_{-}$are in the remaining variables boundary values of functions analytic

1 At this point the proof breaks down in fewer than four dimensions 
in the forward tube $\mathscr{T}_{+}=\left\{\operatorname{Im}\left(x_{2}-x_{1}\right) \in V_{+}, \ldots, \quad \operatorname{Im}\left(Y-x_{\alpha-1}\right) \in V_{+}\right.$, $\left.\operatorname{Im}\left(x_{\alpha+2}-Y\right) \in V_{+}, \operatorname{Im}\left(x_{a+3}-x_{\alpha+2}\right) \in V_{+}, \ldots\right\}$. This follows as usual from the spectral condition, treating e.g. $\int d^{4} y \varphi(y) \psi_{i_{\alpha}}\left(Y-\frac{1}{2} y\right) \psi_{i_{\alpha+1}}\left(Y+\frac{1}{2} y\right)$ like a single field. The same is then true for the difference $\Delta=K_{+}-K_{-}$. But there exist real points $Y, x_{\alpha-1}$, such that $S_{x_{\alpha-1}}$ is space-like to $S_{x_{\alpha}} \cup S_{x_{\alpha+1}}$ for all $y \in \operatorname{supp} \varphi$, and these points form a non-empty open set.

In this set we have

$$
\begin{aligned}
& \Delta\left(\ldots, x_{\alpha-1}, Y, y, x_{\alpha+2}, \ldots\right) \\
& \quad= \pm \Delta\left(\ldots, Y, y, x_{\alpha-1}, x_{\alpha+2}, \ldots\right) \\
& \quad= \pm\left[W^{T}\left(\ldots, x_{\alpha}, x_{\alpha+1}, x_{\alpha-1}, x_{\alpha+2}, \ldots\right) \mp W^{T}\left(\ldots, x_{\alpha+1}, x_{\alpha}, x_{\alpha-1}, x_{\alpha+2}, \ldots\right)\right] \\
& \quad=0
\end{aligned}
$$

by the inductive assumption. By analytic continuation this relation holds everywhere: $W^{T}$ is invariant, up to a sign, under exchange of $x_{\alpha}$ and $x_{\alpha+1}$, hence under all permutations of $x_{1}, \ldots, x_{\alpha+1}$.

As a consequence of this result we have

$$
\tilde{W}^{T}\left(p_{1}, \ldots, p_{n}\right)= \pm \tilde{W}^{T}\left(p_{2}, \ldots, p_{n}, p_{1}\right) .
$$

But the left-hand side has its support contained in $p_{1} \in \bar{V}_{+}$, the right-hand side in $p_{1} \in \bar{V}_{-}$, and a point support at $p_{1}=0$ is forbidden by the cluster property. Hence $\tilde{W}^{T}\left(p_{1}, \ldots, p_{n}\right)=0$.

\section{References}

1. Mandelstam, S.: Quantum electrodynamics without potentials. Ann. Phys. (NY) 19, 1 (1962)

2. Buchholz, D., Fredenhagen, K. : Locality and the structure of particle states. Commun. Math. Phys. 84, 1 (1982)

Fredenhagen, K.: On the existence of antiparticles. Commun. Math. Phys. 79, 141 (1981)

Buchholz, D.: The physical state space of QED. Commun. Math. Phys. 85, 49 (1982)

3. Sarker, A.Q.: On the Green's function of gauge-independent theory of quantum electrodynamics. Ann. Phys. (NY) 24, 19 (1963)

Bialynicki-Birula, I.: Bull. Acad. Polon. Sci. 11, 135 (1963)

Mandelstam, S.: Feynman Rules for electromagnetic and Yang-Mills fields from the gaugeindependent field theoretic formalism. Phys. Rev. 175, 1580 (1968)

4. Streater, R.F., Wightman, A.S. : PCT, spin \& statistics, and all that. New York: Benjamin 1964 Jost, R.: The general theory of quantized fields. Providence, RI: American Mathematical Society 1965

5. Jost, R. : Lectures on field theory and the many-body problem. Caianiello, E.R. (ed.). New York: Academic Press 1961

Federbush, P.G., Johnson, K.A.: Uniqueness property of the twofold vacuum expectation value. Phys. Rev. 120, 1926 (1960)

Pohlmeyer, K. : The Jost-Schroer theorem for zero-mass fields. Commun. Math. Phys. 12, 204 (1969)

6. Streater, R.F., Wightman, A.S.: Loc. cit., Sect. 2-3

7. Streater, R.F., Wightman, A.S. : Loc. cit., Sect. 2-5

8. Bochner, S., Martin, W.T.: Several complex variables, Chap. IV, Sect. 2. Princeton, NJ : Princeton University Press 1948

Communicated by K. Osterwalder 